\title{
How Pitfalls during Drug Quantitation by Mass Spectrometry May Affect the Variability of Pharmacokinetic Data during a Bioequivalence Trial
}

\section{Marcelín-Jiménez G* \\ Global Bioanalytical Consulting, 03200 Mexico City, Mexico}

The majority of the time, during the performance of a bioequivalence trial, clinicians exercise extreme care in the selection of volunteers in order to obtain a homogeneous population, and control several sources of variation, such as diet, fasting period, hydration, and drug administration (among others), to the extent possible, to limit inter- and intra-individual variability and to achieve the statistical power required for the assay. In addition, monitoring staffs is charged with guaranteeing compliance with good clinical practices [1].

In addition, and at the end of the study, some sponsors and Contract Research Organizations (CRO) re-check the databases and re-run the statistics to ensure completely the correct calculation of the pharmacokinetic data. But, what had occurred during the trial's midterm that is, during the bioanalytical phase?

It is known that all regulatory agencies request from pharmaceutical laboratories a fully validated method that certifies reasonable reliability of the quantitative process; however, there are some difficult-tomake-evident analytical situations that imply a deep knowledge of Biochemistry, Physicochemistry, Pharmacology, chromatography, and statistics for the Mass Spectrometrist [2]. Otherwise, the pitfalls that arise will contribute to variability of the results that are not attributable to the population's genetic background, the pharmaceutical formulation, or the clinical conduction of the assay.

The first case considers the presence of endogenous isobaric compounds. During the development of a bioanalytical method for UltraPerformance Liquid Chromatography-Tandem Mass Spectrometer (UPLC-MS/MS) for quantitation of Fosfomycin in human plasma, and according to previous authors, mass spectrometer conditions were set at negative electrospray detection and Fosfomycin was set at 137.08>79.08 $\mathrm{m} / \mathrm{z}^{-1}$. However, a highly fluctuating signal appeared in the Fosfomycin channel, independently of the amount of blank plasma batches analyzed during selectivity. After an exhaustive review of the literature, it appears that phosphoenolpyruvate (a glycolysis by-product) undergoes a rearrangement that yields an ion product of the same weight as that of the ion product of Fosfomycin (and that co-elutes with the antibiotic). The strategy for this case was to change the ion product, settling on a less intense but stable fragment at $62.9 \mathrm{~m} / \mathrm{z}^{-1}$, allowing for quantitation of samples with precision and accuracy, independently of the glycolyticstate of each volunteer along the day [3].

A second situation comprises cross-talking between analyte and internal standard channels. Sometimes, when the use of the deuterated drug as internal standard is not possible (commercially unavailable, very expensive, etc.), the analyst chooses a closely related molecule and attempts the co-elution of such a molecule during chromatography in order to compensate for possible oscillations during ionization. During the analysis of macrolides, such as Azithromycin (MW 748.88) and Clarithromycin (MW 747.95) by multiple-reaction monitoring, the selection of one of these molecules as the internal standard of the other may cause highly variable results. This is due to that both molecules share quite similar patterns of fragmentation, and the signal of one transition contributes non-specifically to the channel of the other molecule. Moreover, during analysis of Erythromycin (a mixture of pharmacologically active Erythromycin A and its B, C, D, and E isomers as impurities), cross-talking into the same channel of the main analyte is possible. Thus, good chromatographic resolution is usually the solution to the problem [4].

The third bioanalytical challenge is to avoid metabolite re-conversion into parent drug during ionization. This might be controlled if the metabolites are included during development and validation assays (however, this is not possible). An example of the first case is the remethylation of demethylated metabolites during ionization, obviously in the presence of methanol as part of the chromatographic mobile phase. This reaction does not evolve constantly and will take place more probably in samples with high levels of the metabolite. Re-conversion of $\mathrm{N}$-desmethyl-clindamycin into Clindamycin is one example. The use of acetonitrile instead of methanol limits the risk of this reaction [5].

The second opportunity for metabolite re-conversion is due to Collision-Induced Dissociation (CID) in the gas-phase, which gives rise to glucuronide hydrolysis. Some drugs, such as Tizoxanide (active metabolite of pro-drug Nitazoxanide) and Sildenafil, are conjugated in the liver with glucuronic acid to facilitate their excretion. Whether both the analyte and its glucuronide are co-extracted during sample processing, this accompanied by chromatography, glucuronide hydrolysis would contribute to the signal of the main analyte. This phenomenon can be evidenced (and prevented) by increasing the chromatographic capacity factor $\left(\mathrm{k}^{\prime}\right)$ of the main analyte; two peaks in the same channel with different retention times (the first corresponding to the drug delivered by CID, and the second, to the non-metabolized drug) should be observed.

In sum, the bioanalytical method is a keystone for obtaining accurate and reliable pharmacokinetics, and the contribution of extractive procedures and instrumentation to data variability must be delimited.

\section{References}

1. Li L, Chen X, Dai X, Chen H, Zhong D (2007) Rapid and selective liquid chromatographic/tandem mass spectrometric method for the determination of Fosfomycin in human plasma. J Chromatogr B 856: 171-177.

2. Pérez DS, Soraci AL, Diéguez SN, Tapia MO (2011) Determination and withdrawal time of Fosfomycin in chicken muscle, liver and kidney. Int $\mathrm{J}$ Poult Sci 10: 644-655.

*Corresponding author: Gabriel Marcelín-Jiménez, Ph.D., Global Bioanalytica Consulting, S.C., Av. Insurgentes Sur No. 1188, Dep. 203, Col. Tlacoquemécatl, Del. Benito Juárez, 03200 Mexico City, Mexico, Tel: 52-55-9127 0111; E-mail: gabmarcelin@gbioanalytical.com.mx

Received May 18, 2017; Accepted May 29, 2017; Published June 09, 2017

Citation: Marcelín-Jiménez G (2017) How Pitfalls during Drug Quantitation by Mass Spectrometry May Affect the Variability of Pharmacokinetic Data during a Bioequivalence Trial. J Bioequiv Availab 9: 430-431. doi: 10.4172/jbb.1000337

Copyright: @ 2017 Marcelín-Jiménez G. This is an open-access article distributed under the terms of the Creative Commons Attribution License, which permits unrestricted use, distribution, and reproduction in any medium, provided the original author and source are credited. 
Citation: Marcelín-Jiménez G (2017) How Pitfalls during Drug Quantitation by Mass Spectrometry May Affect the Variability of Pharmacokinetic Data during a Bioequivalence Trial. J Bioequiv Availab 9: 430-431. doi: 10.4172/jbb.1000337

3. Mess JN, Bérubé ER, Furtado M, Garofolo F (2011) A practical approach to reduce interferences due to in-source collision-induced dissociation of acylglucuronides in LC-MS/MS. Bioanalysis 3: 1741-1751.

4. Contreras Z, Rivera E, Moreno AP, Ramírez San-Juan E, Hernández ZS, et al. (2014) Development of a UPLC-MS/MS method for the quantification of
Sildenafil by DBS, and its use on pediatric pulmonary hypertension. Bioanalysis 6: 2815-2824.

5. Woolf E, McDougall S, Fast D, Andraus M, Barfield M, et al. (2014) Small molecule specific run acceptance, specific assay operation, and chromatographic run quality assessment: recommendation for best practices and harmonization from the global bioanalysis consortium harmonization teams. AAPS J 16: 885-893. 